ARTICLE

Received 13 Sep 2013 | Accepted 4 May 2014 | Published 4 Jun $2014 \quad$ DOl: 10.1038/ncomms5033

\title{
Expanded graphite as superior anode for sodium-ion batteries
}

\author{
Yang Wen ${ }^{1, \star}$, Kai He ${ }^{2, \star}$, Yujie Zhu ${ }^{1, \star}$, Fudong Han ${ }^{1}$, Yunhua $\mathrm{Xu}^{1}$, Isamu Matsuda ${ }^{3}$, Yoshitaka Ishii ${ }^{3,4}$, \\ John Cumings ${ }^{2} \&$ Chunsheng Wang ${ }^{1}$
}

Graphite, as the most common anode for commercial Li-ion batteries, has been reported to have a very low capacity when used as a Na-ion battery anode. It is well known that electrochemical insertion of $\mathrm{Na}^{+}$into graphite is significantly hindered by the insufficient interlayer spacing. Here we report expanded graphite as a $\mathrm{Na}$-ion battery anode. Prepared through a process of oxidation and partial reduction on graphite, expanded graphite has an enlarged interlayer lattice distance of $4.3 \AA$ yet retains an analogous long-range-ordered layered structure to graphite. In situ transmission electron microscopy has demonstrated that the Na-ion can be reversibly inserted into and extracted from expanded graphite. Galvanostatic studies show that expanded graphite can deliver a high reversible capacity of $284 \mathrm{mAhg}^{-1}$ at a current density of $20 \mathrm{mAg}^{-1}$, maintain a capacity of $184 \mathrm{mAhg}^{-1}$ at $100 \mathrm{mAg}^{-1}$, and retain $73.92 \%$ of its capacity after 2,000 cycles.

\footnotetext{
${ }^{1}$ Department of Chemical and Biomolecular Engineering, University of Maryland, College Park, Maryland 20742, USA. ${ }^{2}$ Department of Materials Science and Engineering, University of Maryland, College Park, Maryland 20742, USA. ${ }^{3}$ Department of Chemistry, University of Illinois at Chicago, Chicago, Illinois 60607, USA. ${ }^{4}$ Center for Structural Biology, University of Illinois at Chicago, Chicago, Illinois 60607, USA. ${ }^{*}$ These authors contributed equally to the work. Correspondence and requests for materials should be addressed to C.W. (email: cswang@umd.edu) or to J.C. (email: cumings@umd.edu).
} 
T he Li-ion battery (LIB) industry has been continuously expanding over the last three decades, mostly because of the steadily increasing demands of a growing market for portable electronic devices. However, as demand emerges for hybrid and electric vehicles, and as emphasis shifts to the power grid and other large-scale applications, the limits of the natural abundance and uneven global distribution of lithium-containing precursors present unavoidable challenges to industry. For such large-scale applications, a low-cost and sustainable supply-chain is critical. As an alternative, Na-ion batteries (NIBs) have recently drawn significant attention because, unlike $\mathrm{Li}, \mathrm{Na}$ is a ubiquitous and earth-abundant element. NIBs were originally developed in the late 1980s, in approximately the same time period as LIBs ${ }^{1-3}$, and the demand for large-scale energy storage for grid applications has recently revived the interest. Most of the recent research on NIB electrode materials has focused mainly on cathodes, but here we concentrate instead on a possible new anode material.

Since $\mathrm{Li}$ and $\mathrm{Na}$ share common properties as alkali metals, it is sometimes assumed to be convenient to transfer the insights gained from the science of LIBs to NIBs. However, $\mathrm{Na}^{+}$has a larger radius than $\mathrm{Li}^{+}$, which directly affects the mass transport and storage in the electrochemical process. It makes many of the superior LIB anode materials, such as graphite, unsuitable for NIBs. Graphite, as the most common anode for commercial LIBs, has a long-range-ordered layered structure, and $\mathrm{Li}^{+}$can readily electrochemically intercalate between the graphite layers. The intercalated material has a high theoretical reversible capability of $372 \mathrm{mAh} \mathrm{g}^{-1}$ and long cycle life. However, the electrochemical sodiation/desodiation capacity of graphite is $<35 \mathrm{mAhg}^{-1}$ (refs 4,5). As demonstrated by recent theoretical calculations, the interlayer distance of graphite $(\sim 0.34 \mathrm{~nm})$ is too small to accommodate the large $\mathrm{Na}^{+}$ion (Fig. 1a), and a minimum interlayer distance of $0.37 \mathrm{~nm}$ is believed to be required for $\mathrm{Na}^{+}$ insertion ${ }^{6}$.

A variety of carbon materials, such as carbon with different structures (soft carbons ${ }^{5}$ and hard carbons $s^{5,7,8}$ ), different compositions (hydrogen-contained carbons $s^{5}$ ) and different morphologies (carbon nanotubes ${ }^{6}$, porous carbons ${ }^{9}$, hollow carbon nanoparticles ${ }^{10}$ and reduced graphene oxides ${ }^{11}$ ), have been investigated as anodes for NIB in previous studies. Most of these carbon-based materials consist of few-layer-stacked graphite nanocrystallites with large interlayer distances in the range of $0.37-0.40 \mathrm{~nm}$ (ref. 6). These localized nanocrystallites leave many disordered nanovoids within the materials ${ }^{5,6,9-12}$, and it is believed that the $\mathrm{Na}^{+}$ions can reversibly insert into the graphite nanocrystallites and the nanovoids in these carbon materials. $\mathrm{Na}^{+}$insertion in nanocrystallite usually occurs in a wide potential range from 1.5 to $0.1 \mathrm{~V}$ (versus $\mathrm{Na} / \mathrm{Na}^{+}$), whereas the $\mathrm{Na}^{+}$filling into nanovoids happens in a low and narrow potential range of $0.1-0.0 \mathrm{~V}$ (versus $\left.\mathrm{Na} / \mathrm{Na}^{+}\right)^{5,6,9-12}$. In these two mechanisms, the interlayer insertion reaction would be preferred, as the pore-filling reaction occurs only at low potentials, where it can be accompanied by Na plating during fast charging, causing $\mathrm{Na}$ loss and, more severely, battery failure due to electrical shorting. Certain conclusions can be drawn from these studies, and it is apparent that materials with long-range-ordered layered structures, larger interlayer distances and low porosity are desired.

Here we report expanded graphite (EG) as a superior NIB anode material. EG is a graphite-derived material formed by a two-step oxidation-reduction process that retains the long-rangeordered layered structure of graphite, yielding a generally large interlayer distance $(>0.34 \mathrm{~nm}$; Fig. $1 \mathrm{~b}, \mathrm{c})$. These features provide favourable conditions for electrochemical intercalation of $\mathrm{Na}^{+}$ ions. Moreover, the interlayer spacing of EG can be manipulated by controlled oxidation and reduction processing, which positions EG as a promising anode material for NIBs. We report here that $\mathrm{Na}^{+}$indeed can reversibly insert into and extract from the EG, with an interlayer distance of $0.43 \mathrm{~nm}$. The sodiation- and desodiation-induced microstructure changes of EG are dynamically captured during real-time imaging at the atomic scale using in situ high-resolution transmission electron microscopy (HRTEM). Electrochemical tests show that the EG can provide a high reversible capacity of $284 \mathrm{mAh} \mathrm{g}^{-1}$ at a current density of $20 \mathrm{mAg}^{-1} ; 184 \mathrm{mAhg}^{-1}$ at a current density of $100 \mathrm{mAg}^{-1}$; and retain $73.92 \%$ of the capacity after 2,000 cycles (loss $0.037 \%$ per cycle). Along with the mature and low-cost synthesis technique of graphite anodes for LIBs, EG could be a very promising anode material for the NIBs industry in the near future.

\section{Results}

Material and structural design. EG was synthesized by oxidizing pristine graphite (PG) to become graphite oxide (GO) using modified Hummer's method ${ }^{13}$ and followed by a partial reducing process of GO. The modified Hummer's method is well known as an oxidization method to form GO in the graphene synthesis technique ${ }^{11}$. In a typical graphene synthesis procedure, the resulting GO is sonicated before (or after) the reduction reaction to peel off the functionalized graphene layers from graphite and acquire single-layer or few-layer graphenes. In our synthesis, we intentionally omitted the sonication step to avoid the damage caused by sonication and maintain the long-rangeordered layered structure. More experimental details are provided in Methods section.

The crystal structures of PG, GO and two EG samples (EG-1 h and EG-5h with $1 \mathrm{~h} / 5 \mathrm{~h}$ denoting the thermal reduction period applied on GO to obtain the sample) were characterized using

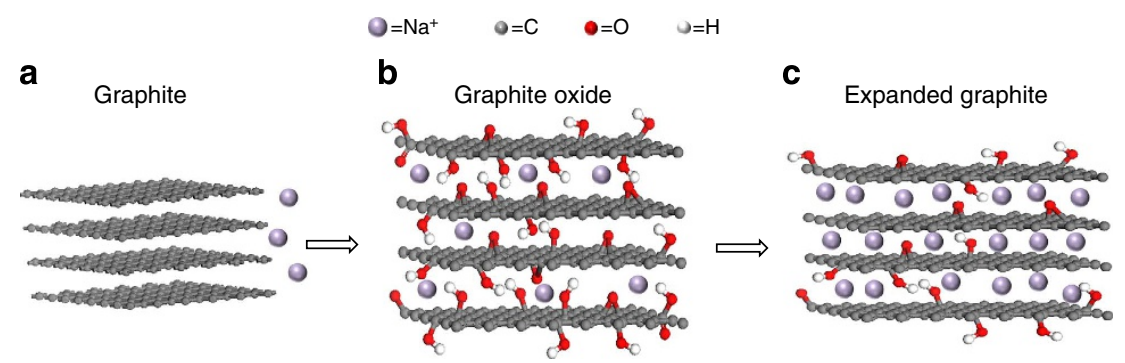

Figure 1 | Schematic illustration of sodium storage in graphite-based materials. (a) $\mathrm{Na}^{+}$cannot be electrochemically intercalated into graphite because of the small interlayer spacing. (b) Electrochemical intercalation of $\mathrm{Na}^{+}$into $\mathrm{GO}$ is enabled by the enlarged interlayer distance because of

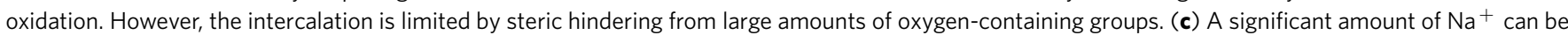
electrochemically intercalated into EG owing to suitable interlayer distance and reduced oxygen-containing groups in the interlayers. 
powder X-ray diffraction (XRD; Supplementary Fig. 1). For the purpose of systematically analysing the reduction time effect, GO was considered as EG-0 h to compare with EG samples (EG-1 h and EG-5h). PG exhibits a sharp peak at $2 \theta \sim 26.6^{\circ}$ (Supplementary Fig. 1), which corresponds to the diffraction of (002) plane with an interlayer distance of $3.36 \AA(\sim 0.34 \mathrm{~nm})$ (refs 14,15). Upon oxidation, the inserted oxygen-containing groups will attach to both sides of the single graphene plane, causing distortion of the interlayer structure ${ }^{16,17}$. The diffraction peak of GO shifts to $2 \theta \sim 13.0^{\circ}$, which indicates an enlarged inter-planar distance. After thermal reduction for $1 \mathrm{~h}$, the diffraction peak of EG-1 h shifts back to $2 \theta \sim 23.0^{\circ}$, yet is still smaller than that in graphite $\left(\sim 26.6^{\circ}\right)$, exhibiting a graphite-like structure with a larger inter-planar distance. As thermal reduction time increases to $5 \mathrm{~h}$, the diffraction peak of EG-5 h shifts to $2 \theta$ $\sim 25.3^{\circ}$, indicating a shrinkage of interlayer distance compared with EG-1 h.

The surface areas of PG, GO and EG samples were characterized by using $\mathrm{N}_{2}$ gas adsorption-desorption. The Brunauer-Emmett-Teller (BET) surface areas are measured to be $13.52 \mathrm{~m}^{2} \mathrm{~g}^{-1}$ for PG, $64.89 \mathrm{~m}^{2} \mathrm{~g}^{-1}$ for GO, $30.22 \mathrm{~m}^{2} \mathrm{~g}^{-1}$ for EG-1 $\mathrm{h}$ and $34.70 \mathrm{~m}^{2} \mathrm{~g}^{-1}$ for EG-5 h. Both EG samples reveal a low surface area, which is much smaller than that of typical graphene/graphene oxide prepared through Hummer's method ${ }^{18}$, indicating low exfoliation degree and good graphite-like stack morphology retention. On the other hand, the low surface area of EGs also implies that only a small amount of pores are introduced during oxidation and thermal reduction process. Different from graphite, the $\mathrm{N}_{2}$ adsorption-desorption isotherm profiles of $\mathrm{GO}$, EG-1 h and EG-5h exhibit no limiting adsorption at high $\mathrm{P} / \mathrm{P}_{0}$ (Supplementary Fig. 2a), revealing a typical character of plate-like particle with slit-shaped pores ${ }^{19}$. The specific surface area of PG used in this study is three times higher than that of previously reported graphite ${ }^{20}$, and is similar to that of the hard carbon (SX114i19 in ref. 21). The EGs' surface areas are similar to the hard carbon (SX114i5 in ref. 21) reported by Dahn and group ${ }^{21}$. The tap densities for PG, GO, EG- $1 \mathrm{~h}$ and $5 \mathrm{~h}$ samples were measured to be $0.72,4.38,2.38$ and $1.88 \mathrm{~g} \mathrm{~cm}^{-3}$ (Supplementary Fig. 2b). The tap densities of EG samples are higher than that of most of reported soft carbons $\left(\sim 0.66-1.26 \mathrm{~g} \mathrm{~cm}^{-3}\right.$; (ref. 22$)$ and hard carbon $\left(\sim 0.50-0.81 \mathrm{~g} \mathrm{~cm}^{-3}\right.$; (refs 22,23$)$, whereas the PG's tap density is similar to the hard carbon ${ }^{21}$. The EG samples were observed to be more condensed than the reported carbon material used in $\mathrm{LIB}^{21}$. The graphitization degree of $\mathrm{PG}, \mathrm{GO}$ and EG materials was determined by Raman spectroscopy. All samples exhibit typical $D$ band (centred at $\sim 1,360 \mathrm{~cm}^{-1}$ ) and $G$ band (centred at $\sim 1,585 \mathrm{~cm}^{-1}$ ) for carbon material ${ }^{24}$. The $\mathrm{D}$ band corresponds to the disordered carbon, whereas the $G$ band represents the typical signal for graphite-like morphology in carbon material. The $I_{\mathrm{D}} / I_{\mathrm{G}}$ intensity ratios for PG, GO, EG-1 h and EG-5 h are 0.22, 0.86, 0.88 and 0.97 (Supplementary Fig. 2c), indicating that EGs partially maintain the graphitic character. The electronic conductivities of PG, GO, EG-1 h and EG-5h were measured to be $461.47,87.65,322.62$ and $363.40 \mathrm{~S} \mathrm{~cm}^{-1}$, respectively (Supplementary Fig. 2d). Both EG samples show much better conductivity than GO (EG- $0 \mathrm{~h}$ ) because of the removal of oxygen-containing groups during thermal reduction.

The structures of PG, GO and EGs were further characterized by TEM. Figure 2 shows HRTEM images of the cross-sectional view for PG, GO, EG-1 h and EG-5h, in which the layered structures of these specimens are well resolved by the alternating bright and dark contrast. The PG displays well-defined layers of long-range order stacking parallel to each other (Fig. 2a). After oxidation, such long-range order is disrupted by massive intercalation of oxygen in the graphite interlayer, forming a more disordered pattern with only localized short-range ordering, as shown in Fig. 2b. However, after partial reduction, the material recovers toward a well-organized morphology, while preserving expanded interlayer distances and the long-range channels suitable for $\mathrm{Na}$ ions transport, as shown in Fig. 2c,d. In addition, the progressive change in interlayer distance is also clearly apparent. The contrast line profiles across five stacking layers at representative locations, indicated by the arrows, are plotted under the corresponding images. The average interlayer spacings are measured to be $\sim 0.34 \mathrm{~nm}$ for PG, $0.61 \mathrm{~nm}$ for GO, $0.43 \mathrm{~nm}$ for EG-1 h and $0.37 \mathrm{~nm}$ for EG-5 h. It is obvious that the distance between PG layers is expanded after the oxidation reactions because of the insertion of oxygen-containing groups, and that it still retains larger than PG after reduction treatments.

Nature and content of oxygen-containing groups in the interlayer. From above XRD and HRTEM results, it is clear that the interlayer distances of PG, GO and EGs are related to the a
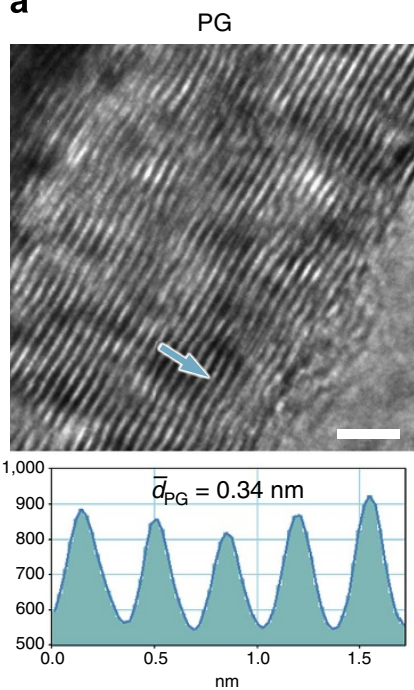

b
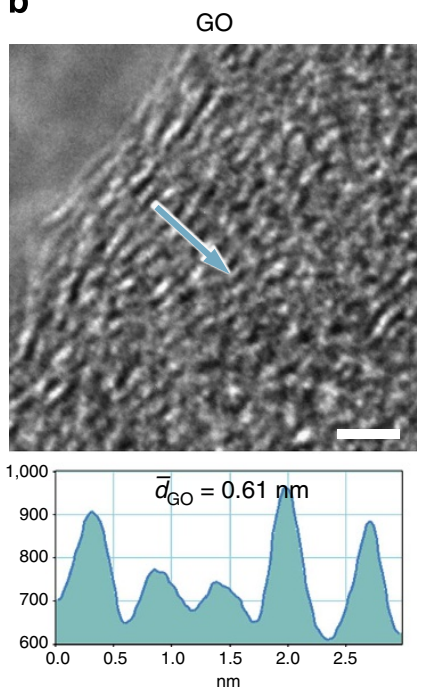

C
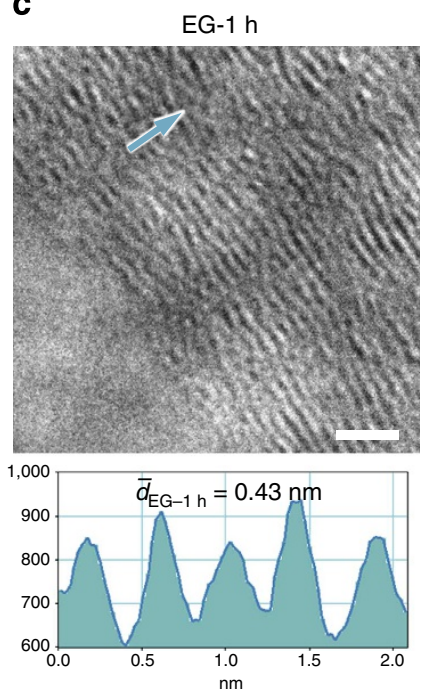

d
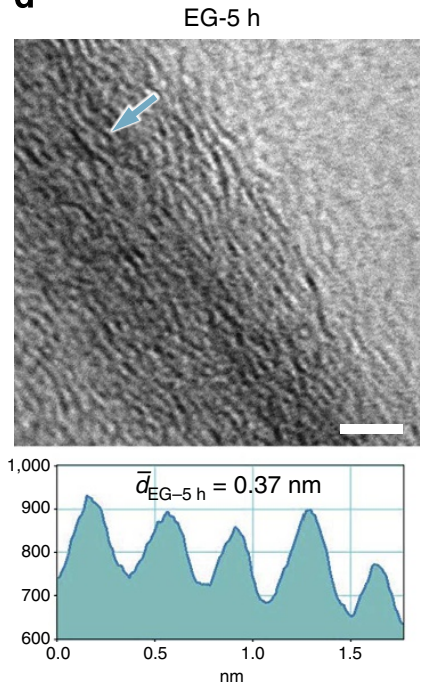

Figure 2 | High-resolution TEM analysis of PG, GO and EG. High-resolution TEM images showing cross-sectional layered structures for (a) PG, (b) GO, (c) EG-1 h and (d) EG-5 h. Scale bars, $2 \mathrm{~nm}$. Contrast profiles along the arrows indicate interlayer spacings of corresponding samples. 
nature and content of oxygen-containing groups in the interlayer. So, the nature and amount of these oxygen-containing groups in PG, GO and EG samples (EG-1 h and EG-5 h) were investigated using X-ray photoelectron spectroscopy (XPS), and the results are shown in Fig. 3a-d, where C $1 \mathrm{~s}$ peak presents at $\sim 284.8 \mathrm{eV}$ and $\mathrm{O} 1 \mathrm{~s}$ peak presents at $\sim 533.6 \mathrm{eV}$. Figure $3 \mathrm{e}$ illustrates the $\mathrm{C} / \mathrm{O}$ ratio calculated from the intensity of $\mathrm{C} 1 \mathrm{~s}$ and $\mathrm{O} 1 \mathrm{~s}$ peaks for different samples. PG contains a very small amount of oxygen (3.56\%) (Fig. 3a). Upon oxidation, the intensity of $\mathrm{O} 1 \mathrm{~s}$ peak increases significantly (Fig. $3 \mathrm{~b}$ ) and the oxygen component reaches to $33.82 \%$, indicating a high oxidation state. After $1 \mathrm{~h}$ thermal reduction in argon (Ar), the oxygen content of EG-1 h sample reduces to $\sim 10 \%$ (Fig. $3 \mathrm{c}$ ), which is much less than that in GO, but it is still three times higher than that in PG. The residual $10 \%$ oxygen-containing groups maintain the interlayer distance at $\sim 0.43 \mathrm{~nm}$ (Fig. $3 \mathrm{c}$ ). As the reduction time increases from 1 to $5 \mathrm{~h}$, the oxygen content further drops to $8 \%$ in EG-5h.
The $2 \%$ decrease in $\mathrm{O}$ component was attributed to further loss of functional groups between graphene layers, resulting in some shrinkage of the interlayer distance, as evidenced by the HRTEM results in Fig. 2 d.

The nature of oxygen-containing functional groups was further analysed by the high-resolution C $1 \mathrm{~s}$ scans (Supplementary Fig. 3). The minor $\mathrm{O}$ component in $\mathrm{PG}$ exists in the form of $\mathrm{C}-\mathrm{OH}$ or $\mathrm{C}-\mathrm{O}-\mathrm{C}$ groups (Supplementary Fig. 3a), whereas the $\mathrm{O}$ content in $\mathrm{GO}$ mainly exists as $\mathrm{C}-\mathrm{OH}, \mathrm{C}-\mathrm{O}-\mathrm{C}$ and $\mathrm{C}=\mathrm{O}$ components (Supplementary Fig. 3b). After thermal reduction, most of the oxygen-containing groups in $\mathrm{GO}$ are removed. A small amount of residual $\mathrm{O}$ in the interlayer exist as $\mathrm{C}-\mathrm{OH} / \mathrm{C}-\mathrm{O}-$ $\mathrm{C}$ and $\mathrm{C}=\mathrm{O}$ groups in EG-1 $\mathrm{h}$ and EG-5h (Supplementary Fig. 3c,d).

The structure evolution along oxygen-containing groups amount change was further investigated by magic-angle-spinning nuclear magnetic resonance (MAS-NMR) experiment. In the ${ }^{13} \mathrm{C}$



C
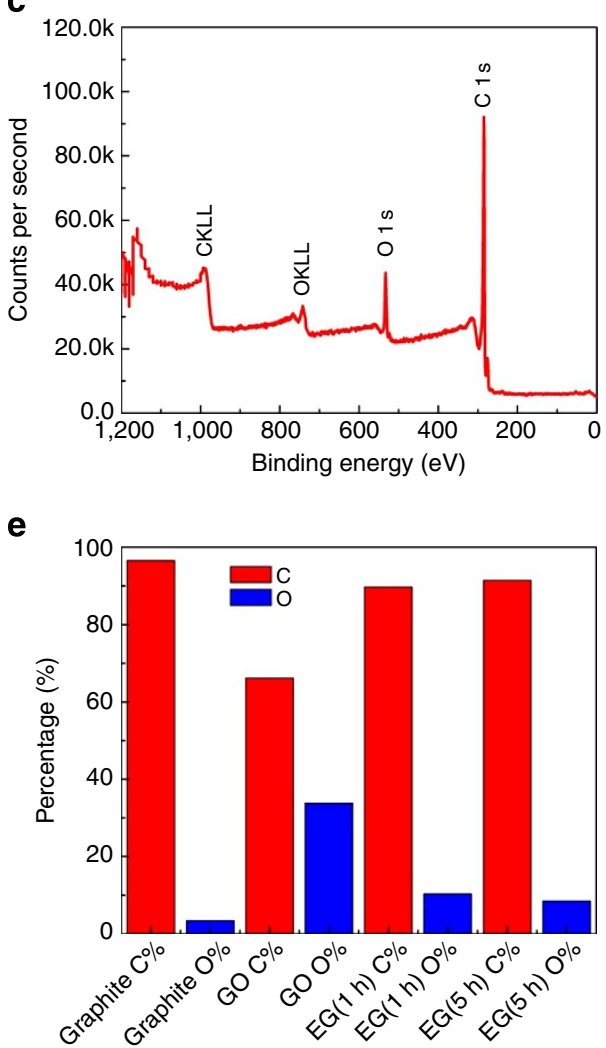

b

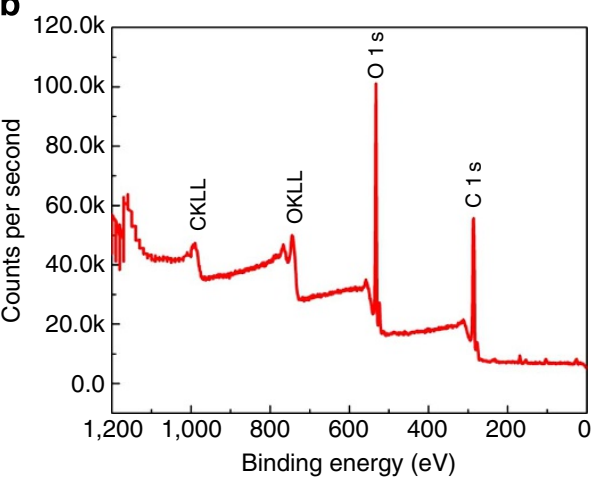

d

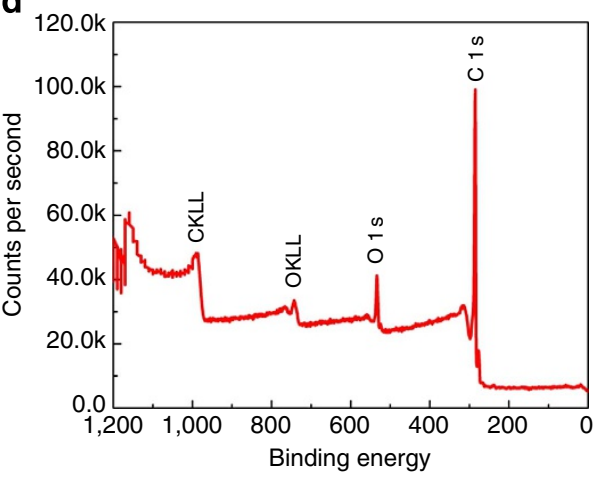

Figure 3 | XPS investigation of the oxygen content. Wide-range XPS spectra of (a) graphite, (b) GO, (c) EG-1h (d) EG-5 h and (e) percentage of C and O element ratio in different samples. 
spectrum of GO (Fig. 4a), three major peaks at 131, 69 and 61 p.p.m. evidence the occurring of the oxidization that is consistent to the previous study ${ }^{25,26}$. The weak peaks at $\sim 165$ and $\sim 190$ p.p.m. (indicated by blue arrows) can be assigned to $-\mathrm{CO}_{2}-$ and $\mathrm{C}=\mathrm{O}^{27}$, consistent with the XPS measurements. The EG-1 $\mathrm{h}$ and EG-5 h samples show a single broader major peak at 117 p.p.m. (Fig. 4b) and 116 p.p.m. (Fig. 4c) with another broad shoulder peak at $\sim 170$ p.p.m. The observed ${ }^{13} \mathrm{C}$ shifts are consistent with those for $\mathrm{sp}^{2}$ carbons without oxidized sites $(\sim 120$ p.p.m.) such as graphene or graphite, suggesting the restoration of $\mathrm{sp}^{2}$ conjugation network. The change in the ${ }^{13} \mathrm{C}$ shift position for the $\mathrm{sp}^{2}$ species from $\sim 130$ p.p.m. in $\mathrm{GO}$ to $\sim 120$ p.p.m. in EG reflects the removal of electron-withdrawing oxygen-containing groups by the reduction. No noticeable peaks were observed either at 60 or 70 p.p.m., although a weak peak for the $\mathrm{C}-\mathrm{OH}$ group may overlap with a tail of the $\mathrm{sp}^{2}$ carbons at 120 p.p.m. The results suggest that thermal reduction for $1 \mathrm{~h}$ is sufficient to restore the $\mathrm{sp}^{2}$ network in the EG sample. Based on the integral intensities of the spectra at $40-80$ p.p.m. region in Fig. 4 b,c, these oxidized species should constitute $\sim 3-8 \%$ of the carbon. This portion of the carbon can be attributed to the $\mathrm{C}-\mathrm{OH}$ or epoxide from graphene plane that is highly related to the interlayer spacing. The broad shoulder peak at $\sim 170$ p.p.m. may be attributed minor species (such as $\mathrm{C}=\mathrm{O}$ and $-\mathrm{CO}_{2} \mathrm{H}$ species) that could be found around the edges/defects. Minor discrepancies from the XRD results may be attributed to the oxygen species at the edges/defects.

To identify $\mathrm{C}-\mathrm{OH}$ groups and other ${ }^{1} \mathrm{H}$-containing species in the GO and EG samples, ${ }^{13} \mathrm{C}^{-1} \mathrm{H}$ dipolar dephasing experiments were performed by using a ${ }^{13} \mathrm{C}-{ }^{1} \mathrm{H}$ rotational echo double
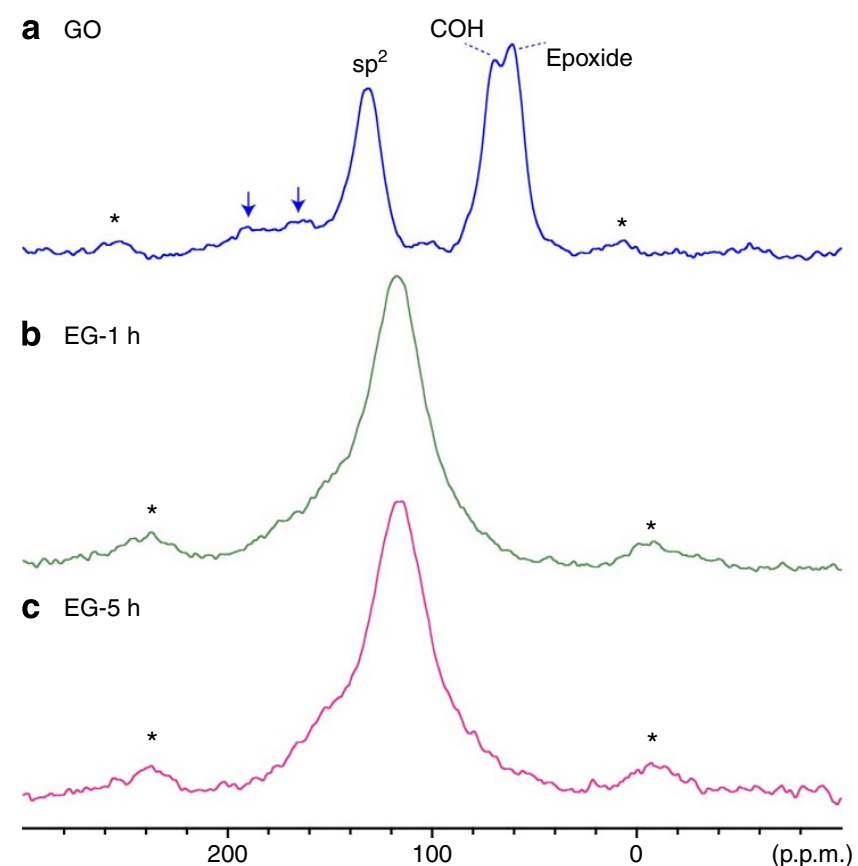

Figure 4 | Solid-state NMR study. 1D ${ }^{13} \mathrm{C}$ MAS solid-state NMR spectra of (a) GO, (b) EG-1h and (c) EG-5 h. All the spectra were obtained at a ${ }^{13} \mathrm{C}$ NMR frequency of $125.78 \mathrm{MHz}$ with $15 \mathrm{kHz}$ MAS for the samples of (a) $33 \mathrm{mg}$, (b) $38 \mathrm{mg}$, (c) $45 \mathrm{mg}$. The ${ }^{13} \mathrm{C}$ MAS spectra were obtained with direct ${ }^{13} \mathrm{C}$ excitation by a $\pi / 2$-pulse followed by a rotor-synchronous echo. The peaks marked by * denote spinning sidebands. The recycle delay was $2 \mathrm{~s}$, and the experimental times were (a) $3 \mathrm{~h}$ and $(\mathbf{b}, \mathbf{c}) 7 \mathrm{~h}$. A Lorentz broadening of $300 \mathrm{~Hz}$ was applied to improve the signal-to-noise ratios. ${ }^{13} \mathrm{C}$ $\mathrm{T}_{1}$ values of the samples were found to be $0.5-0.6 \mathrm{~s}$. The ${ }^{13} \mathrm{C} \pi / 2$-pulse widths are (a) $3.65 \mu \mathrm{s}$ and $(\mathbf{b}, \mathbf{c}) 5.75 \mu \mathrm{s}$. resonance (REDOR) pulse sequence. It is notable that for GO sample, the signal for $\mathrm{C}-\mathrm{OH}$ was mildly dephased (Supplementary Fig. 4). The difference between non-dephased spectrum (Supplementary Fig. 4a) and dephased spectrum (Supplementary Fig. $4 \mathrm{~b}$ ) clearly shows that the signal intensities for the $\mathrm{C}-\mathrm{OH}$ and epoxide groups are reduced by about $20 \%$ and $13 \%$, respectively, whereas the intensity for the $\mathrm{sp}^{2}$ group is reduced by only $6 \%$. The strongest dephasing is a direct evidence of the existent of massive oxygen-containing species, especially the $\mathrm{C}-\mathrm{OH}$, in $\mathrm{GO}$.

This approach is adopted to analyse EG-1 h and EG-5h. Supplementary Fig. 5 shows ${ }^{13} \mathrm{C}$ MAS spectra of EG-1h (Supplementary Fig. 5a-c) and EG-5 h (Supplementary Fig. 5d-f) without and with ${ }^{13} \mathrm{C}-{ }^{1} \mathrm{H}$ dipolar dephasing by ${ }^{13} \mathrm{C}-{ }^{1} \mathrm{H}$ REDOR, together with corresponding difference spectra (Supplementary Fig. 5c,f). For the both samples, no notable selective dephasing was observed unlike the above-mentioned experiment for GO. Rather, the peaks corresponding to $\mathrm{sp}^{2}$ group at $\sim 120$ p.p.m. were uniformly dephased for EG-1 h (Supplementary Fig. 5c) and EG$5 \mathrm{~h}$ (Supplementary Fig. $5 \mathrm{f}$ ) by $12 \%$ and $8 \%$, respectively. It is noteworthy that the signal reduction is higher than that was observed for $\mathrm{sp}^{2}$ species in GO. The results indicate that the surface of the restored graphene sheets in EG can be covered by hydrogen-containing species, and these species may be, in part, responsible for expanded interlayer distances. Indeed, that EG-5 $\mathrm{h}$ shows a lower dephasing than EG-1 $\mathrm{h}$, which is consistent with the observed smaller interlayer distance for EG-5 h.

Effect of oxygen content on sodium storage capacity. Theoretical calculations have revealed that when the interlayer spacing increases to $\sim 0.37 \mathrm{~nm}$, the energy barrier for $\mathrm{Na}^{+}$insertion drops to $0.053 \mathrm{eV}$ (ref. 6). This calculation suggests that it is thermodynamically feasible to electrochemically insert $\mathrm{Na}^{+}$into EG-1 h. Although a large interlayer distance is desired for $\mathrm{Na}^{+}$ insertion, too many oxygen-containing groups between graphene layers could reduce the available positions for $\mathrm{Na}^{+}$and slow the $\mathrm{Na}^{+}$transport. The amount of remaining oxygen-containing groups in the interlayer of EG should in principle be optimized to maintain the sufficient interlayer distance and maximize possible positions for Na-ion storage.

The effects of oxygen content on sodiation/desodiation capacity were investigated using galvanostatic charge/discharge at a current density of $20 \mathrm{mAg}^{-1}$. The charge and discharge curves of PG, GO, EG-1 $\mathrm{h}$ and EG-5h at the second cycles are presented in Fig. 5a. Retarded by the small interlayer spacing, PG has a negligible sodium storage capacity of $\sim 13 \mathrm{mAh} \mathrm{g}^{-1}$, which is widely known and has been reported previously ${ }^{28}$. Oxidation of graphite increases the interlayer distance from 0.34 to $0.61 \mathrm{~nm}$, allowing $\mathrm{Na}^{+}$intercalation into interlayer of GO. The GO shows monotonic voltage curves during the whole sodiation-desodiaton process with an increased capacity of $\sim 156 \mathrm{mAh} \mathrm{g}^{-1}$. However, $33.82 \%$ of oxygen-containing groups in the interlayer reduce the amount of possible positions for $\mathrm{Na}^{+}$storage. After $1 \mathrm{~h}$ of thermal reduction, $23.80 \%$ of the oxygen-containing functional groups are removed, allowing more $\mathrm{Na}^{+}$to insert into the carbon interlayer of EG, despite the reduced interlayer distance of EG-1 h as $0.43 \mathrm{~nm}$. EG-1 h can deliver a high reversible $\mathrm{Na}^{+}$capacity of $\sim 300 \mathrm{mAh} \mathrm{g}^{-1}$, which is significantly higher than PG and GO. When the reduction time extends to $5 \mathrm{~h}$, the capacity of EG-5h drops to $\sim 100 \mathrm{mAh} \mathrm{g}^{-1}$. Although similar charge-discharge pattern was observed between EG-1 h and EG-5 h, the capacity delivered by EG-5h decreased significantly, implying that the interlayer distance predominantly controls the $\mathrm{Na}^{+}$storage capacity. The sodiation curve of EGs clearly consists of two stages, that is, monotonic sloping voltage profile between 2.0 and $0.3 \mathrm{~V}$ 
a
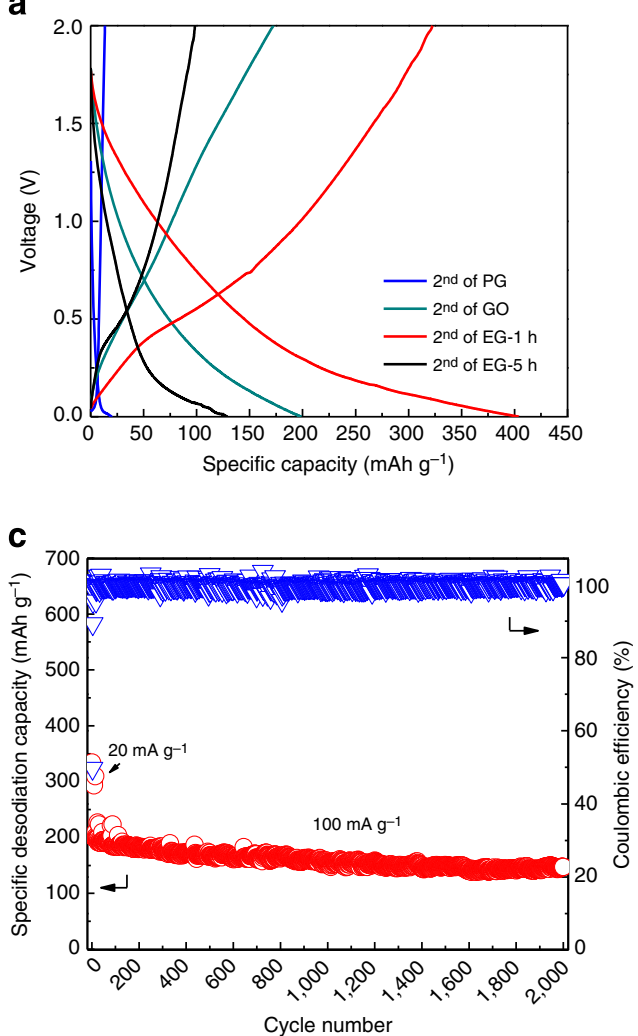

b

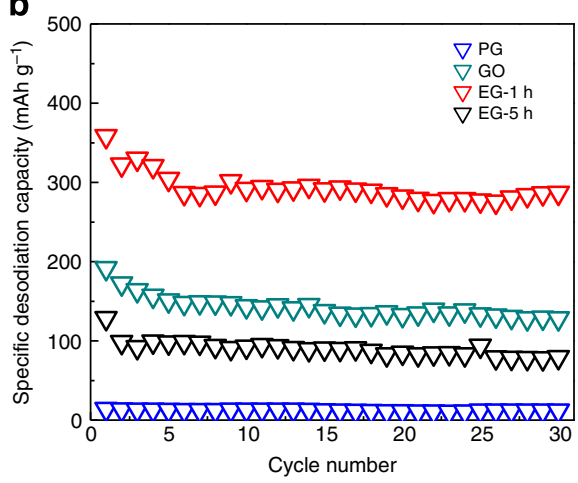

d

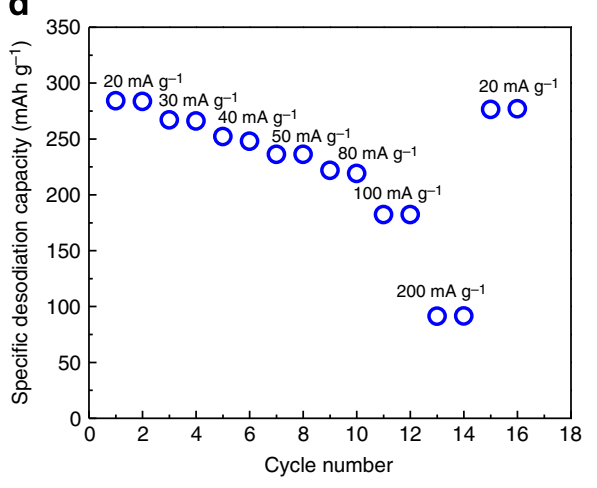

Figure 5 | Electrochemical performances. (a) Charge/discharge curves for the second cycles of PG, GO, EG-1h and EG-5 h at a current density of $20 \mathrm{mAg}^{-1}$. (b) Short-term cycling stability test for PG, GO, EG-1 h and EG-5 h at a current density of $20 \mathrm{mAg}^{-1}$. (c) Long-term cycling stability of EG-1 h. Note that $20 \mathrm{mAg}^{-1}$ was used for the initial 10 cycles. At the 11 th cycle, the current was changed to $100 \mathrm{mAg}^{-1}$ and held constant during the rest of the test. (d) Rate capability test for EG-1h. Note that the cell was pre-cycled at $20 \mathrm{~mA} \mathrm{~g}^{-1}$ for 20 cycles before the rate performance test. Capacities shown in $\mathbf{b}-\mathbf{d}$ are specific desodiation capacities.

(versus $\mathrm{Na} / \mathrm{Na}^{+}$) and an inclined voltage plateau from 0.3 to $0.0 \mathrm{~V}$ (versus $\mathrm{Na} / \mathrm{Na}^{+}$). As reported by previous studies on $\mathrm{Na}$ storage in hard carbon and soft carbon, the monotonic curves occurring in the high voltage region $(>0.3 \mathrm{~V})$ correspond to insertion/extraction of $\mathrm{Na}^{+}$between graphene layers in $\mathrm{EG}^{5,29}$, whereas the low-voltage plateau may represent the $\mathrm{Na}^{+}$ insertion/extraction in the pores/defects ${ }^{5,29}$. The slope in the low-voltage region $(<0.3 \mathrm{~V})$ in desodiation curve could be possibly attributed to hysteresis cause by the interaction between $\mathrm{Na}^{+}$and the residual hydrogen ${ }^{5,30}$. The second stage between $0.3-0.7 \mathrm{~V}$ should be corresponded to the $\mathrm{Na}^{+}$removal from pores, while the slope at high voltage range $(>0.7 \mathrm{~V})$ should be attributed to $\mathrm{Na}^{+}$extraction from graphitic layers.

The EG has long range graphene layer stacked approximately parallel to each other producing relative turbostratic disorder, which is similar to soft carbon ${ }^{5}$. When $\mathrm{Na}$-ion inserts into turbostratically arranged layers, the distribution of sites generates a range of chemical environments, thus a sloping potential curves $^{5}$. However, when the sodium inserts into pores in the EG generated during oxidation/reduction process, there is very little charge transfer from the carbon to the sodium. Therefore, the sodium insertion into/extraction from pores shows a low-voltage plateau ${ }^{5}$. In addition, the existing of oxygen-containing groups such as $-\mathrm{C}=\mathrm{O}$ could also contribute to $\mathrm{Na}^{+}$storage, exhibiting sloping profile at a high voltage range ${ }^{31}$.

As an observed result, charge/discharge capacity initially increases (from PG, GO to EG-1 h), and then decreases (from EG-1 $\mathrm{h}$ to EG-5h) with oxidation and reduction process. The cycling tests of PG, GO, EG-1 h and EG-5 h are shown in Fig. 5b.
All materials show stable cycling performances in the first 30 cycles except the initial few cycles, with EG-1 h exhibiting the highest sodium storage capacity. So, EG-1 h was selected for further electrochemical tests and investigation of sodium storage mechanisms. And thus the term 'EG' that appears in the following sections refers to EG-1 h.

Electrochemical performances of EG. The long-term cycling stability of EG was investigated at a low cycling rate of $20 \mathrm{~mA} \mathrm{~g}^{-1}$ for the first 10 cycles to achieve a full sodiation capacity and then at a high current density of $100 \mathrm{mAg}^{-1}$, as shown in Fig. 5c. EG provides a capacity of $\sim 280 \mathrm{mAhg}^{-1}$ at $20 \mathrm{mAg}^{-1}$ and $\sim 180 \mathrm{mAh} \mathrm{g}^{-1}$ at a high current density of $100 \mathrm{~mA} \mathrm{~g}^{-1}$. The EG demonstrates excellent cycling stability for 2,000 cycles with a very low capacity decay rate of $\sim 0.013 \%$ per cycle from the 11 th cycle to 2,000th cycle. Even though the sodium storage capacity of EG is similar to the reported soft carbon ${ }^{5}$ and hard carbon ${ }^{7,8}$, the capacity retention of EG is much better. The first cycle coulombic efficiency of EG was $\sim 49.53 \%$, and approached $100 \%$ after $\sim 10$ cycles. Such a low first cycle coulombic efficiency was also reported for sodium storage in hard carbons ( $\sim 40-60 \%$; refs 7,8$)$, and it was usually attributed to the formation of solid electrolyte interphase film, which can likely be reduced by (1) changing the electrolyte composition and (2) reducing the defects and voids of EG, both of which have been successfully applied to LIBs.

For the rate capability tests, technologically significant capacities were achieved up to a current density of $200 \mathrm{mAg}^{-1}$. The specific cell was firstly cycled at $20 \mathrm{mAg}^{-1}$ for 20 cycles to 
achieve stable cycling status before the rate capability experiment. As shown in Fig. 5d, EG exhibits a capacity of $284 \mathrm{mAh} \mathrm{g}^{-1}$ at $20 \mathrm{mAg}^{-1} ; 184 \mathrm{mAhg}^{-1}$ at $100 \mathrm{mAg}^{-1} ; 91 \mathrm{mAhg}^{-1}$ at $200 \mathrm{mAg}^{-1}$; and $278 \mathrm{mAhg}^{-1}$ when the current density is changed back to $20 \mathrm{mAg}^{-1}$, demonstrating the stability of EG under a wide range of charge-discharge conditions. The rate capability of EG is modest compared with reported hard carbon ${ }^{8}$, and it could be further improved through nanoengineering to optimize EG's microstructure.

Investigation on the sodium storage mechanism in EG. Cyclic voltammetry was used to analyse the sodium storage mechanism. EG was tested at the scan rates of $0.05,0.1,0.5,1,2,3,5$,
10 and $20 \mathrm{mVs}^{-1}$, as shown in Supplementary Fig. 6a. In Supplementary Fig. 6b,c, a linear relationship was found between the capacity and the reciprocal square root of scan rate, suggesting that the capacity was mainly limited by ion diffusion, similar to most battery electrodes ${ }^{32}$, because capacitive contribution, that is, pseudocapacitance because of surface reaction between $\mathrm{Na}^{+}$and oxygen-containing functional groups (for example, $-\mathrm{C}=\mathrm{O})^{31}$ and double layer charging should be independent of the scan rate ${ }^{33}$. The capacitive contribution was approximately estimated by extrapolating the plot to $V^{-1 / 2}$ QUOTE, and it only contributed to $10 \%$ of the total sodiation capacity (Supplementary Fig. 6a) and 11\% of the total desodiation capacity (Supplementary Fig. 6b) at $0.05 \mathrm{mVs}^{-1}(\sim 0.1 \mathrm{C}$ rate $)$, indicating that the majority of
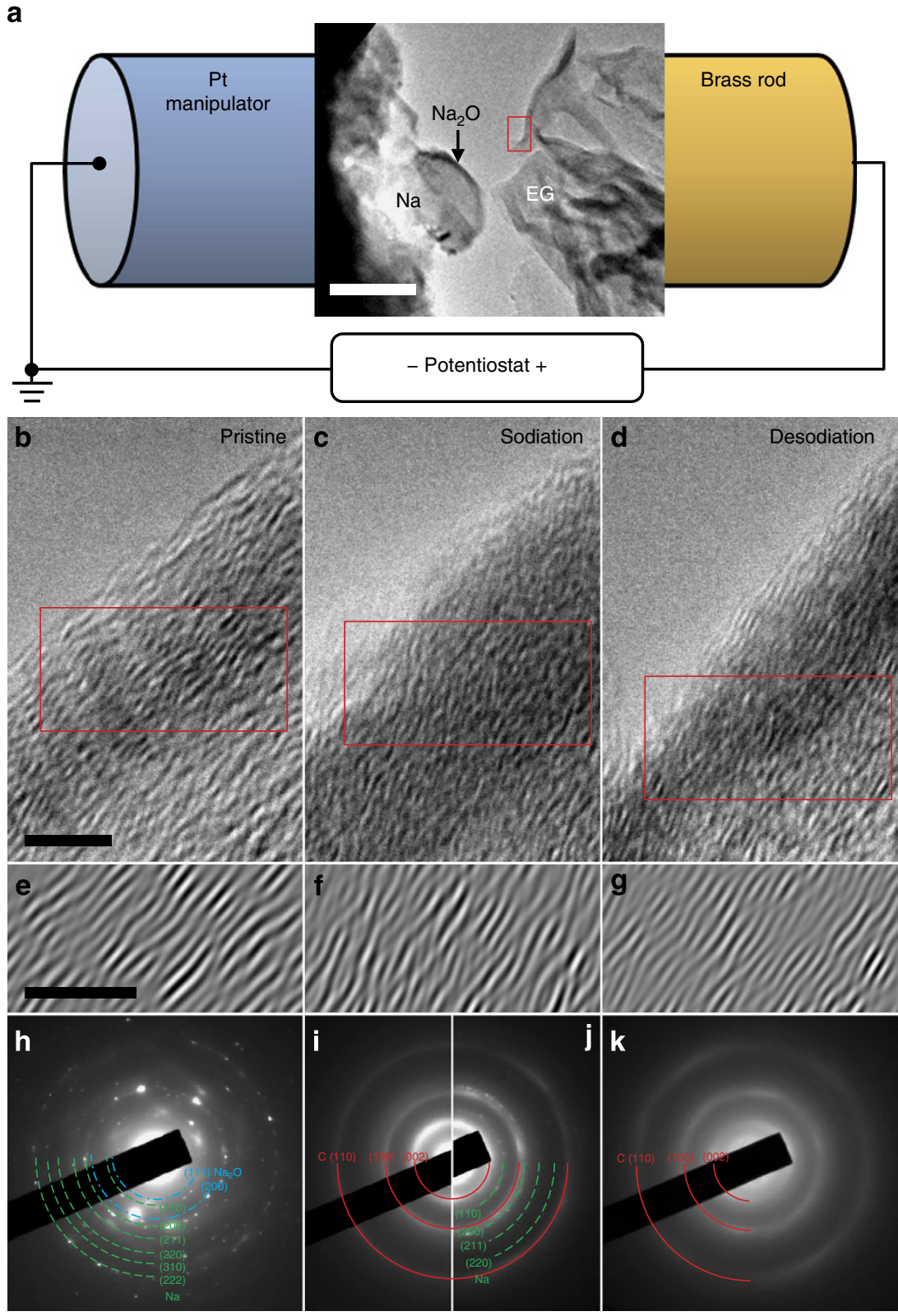

Figure 6 | In situ TEM investigation of sodium storage mechanism in EG-1 h. (a) Schematic illustration of the in situ experimental setup. TEM graph shows EG-1 h on brass rod as the working electrode, $\mathrm{Na}$ metal on Pt manipulator as the counter electrode, and $\mathrm{Na}_{2} \mathrm{O}$ on $\mathrm{Na}$ surface as the solid electrolyte. Scale bar, $200 \mathrm{~nm}$. The boxed region in a corresponds to high-resolution images in b-d. Typical microstructures of (b) pristine EG-1 h, (c) EG-1 h after the first sodiation, and (d) EG-1 h after the first desodiation. Scale bar, $5 \mathrm{~nm}$. (e-g) Filtered TEM close-up images indicating representative microstructural features corresponding to the boxed areas in $\mathbf{b}-\mathbf{d}$, respectively. Scale bar, $5 \mathrm{~nm}$. Electron diffraction patterns from (h) Na source area, (i) EG-1 h at pristine state, (j) EG-1 h after the first sodiation, and (k) EG-1 h after the first desodiation. 
the capacity is due to intercalation and it is highly reversible. This mixed storage mechanism was also observed for lithium storage in soft carbon ${ }^{34}$ in which the lithium storage capacity from intercalation was found to be reversible and stable with cycling ${ }^{34}$. This explains the long and stable cycling performance of EG (Fig. 5c), as majority of the sodium storage capacity in EG is attributed to intercalation as demonstrated by Supplementary Fig. 6b,c.

In situ TEM observation was conducted to provide further insights into the sodium storage mechanism and visualize microstructure evolution of EG during the sodiation and desodiation processes. Figure $6 \mathrm{a}$ illustrates the experimental setup of the NIB in the half-cell configuration, in which the EG glued on a brass rod with conducting adhesive serves as the working electrode, and the $\mathrm{Na}$ metal on a $\mathrm{Pt}$ manipulator as the counter electrode. A layer of naturally formed $\mathrm{Na}_{2} \mathrm{O}$ on the surface of $\mathrm{Na}$ source because of residual oxygen in the TEM vacuum is confirmed by the electron diffraction pattern from this area (Fig. 6h) and serves as the solid electrolyte for $\mathrm{Na}$-ion transportation. The red boxed region at the curled edge of the graphite bulk was selected for high-resolution observations during the electrochemical reactions, and the corresponding close-up TEM morphologies for pristine EG, sodiated EG and desodiated EG are shown in Fig. 6b-d, respectively. To extract the relevant contrast, boxed areas at the approximate positions are replotted to display the representative features after filtering out high-frequency noise, as shown below the corresponding TEM images in Fig. $6 \mathrm{e}-\mathrm{g}$, respectively. The pristine EG displays a microstructure with well-aligned and long-range-ordered graphite layers (Fig. 6b,e) and contains large-scale pure graphitic structure with large interlayer distance (Fig. 6i). After contacting the surface $\mathrm{Na}_{2} \mathrm{O}$ electrolyte to the $\mathrm{EG}$ and applying a negative voltage of $-3 \mathrm{~V}$ on the $\mathrm{EG}$ electrode (versus $\mathrm{Na}$ metal), the sodiation process initiates. As the $\mathrm{Na}$ ions migrate into the EG, the shape of the EG changes by tilting or distorting to accommodate the large-scale morphology changes. It is obvious that the microstructural disorder is complicated and more ripplelike features appear in the graphite-layered pattern (Fig. 6c,f). The electron diffraction pattern taken after the first sodiation (Fig. 6j) indicates that an amount of $\mathrm{Na}^{+}$has been accommodated into the EG. The sodiation-induced structure change was also observed by ex situ Raman spectra (Supplementary Fig. 7). Compared with pristine EG, the intensity ratio between $\mathrm{D}$ band and $\mathrm{G}$ band becomes more pronounced for sodiated EG, indicating the increment in structure randomness after sodiation.

When a positive voltage is applied on the EG against the $\mathrm{Na}$ electrode, the desodiation process begins. As $\mathrm{Na}$ ions migrate out of the EG anode, the microstructure of desodiated EG is reversibly recovered to a parallel layered pattern with long-range ordering (Fig. 6d,g), and the residual $\mathrm{Na}$ ions in EG are found to be negligible as demonstrated by the disappearance of diffraction spots in Fig. 6k. It is also noted that, regardless of microstructural reorganization, the layered structure of the material does not exhibit large-scale distortion throughout the entire reaction, which provides a constant transport space for $\mathrm{Na}$ ions. Although the clear reaction front of these electrochemical reactions was not directly imaged, owing to insufficient volume change, the actual microstructural distortion and restoration associated with the sodiation and desodiation processes have been readily captured by in situ TEM. It is of great importance that such microstructural evolutions are reversible and reproducible without any mechanical or electrochemical breakdown for the observed six charge/discharge cycles (Supplementary Fig. 8, and would also be expected for further cycles according to the excellent cycling performance). Evident from the in situ TEM results, EG as the NIB anode material exhibits excellent performance attributed to the advantages of the unique and robust long-range layered structures. The in situ TEM results are consistent with postmortem HRTEM study of after-cycled EG assembled in coin cells using liquid electrolyte. As shown in the HRTEM image in Supplementary Fig. 9, after 150 sodiation-desodiation cycles, the EG still exhibits a long-lived layered structure with interlayer distance remaining at $0.42 \mathrm{~nm}$, whereas the other local disordered contrast could be caused by effects of interaction with $\mathrm{Na}^{+}$ morphologies.

\section{Discussion}

We have successfully developed EG with a long-range-ordered layered structure for NIB anodes. Different from other carbonaceous NIB anodes, EG stores $\mathrm{Na}^{+}$mostly by interlayer intercalation, which has been confirmed by cyclic voltammetry and in situ TEM observations through reversible interlayer expansion/shrinkage of the EG material during the sodiation and desodiation. The robustness of the long-range-ordered layered structure of EG during sodiation/desodiation has also been revealed by reproducible interlayer changes during multiple charge/discharge processes during in situ TEM observation. For practical NIB applications, EG can deliver a high reversible capacity of $284 \mathrm{~mA} \mathrm{~g}^{-1}$ at a current density of $20 \mathrm{~mA} \mathrm{~g}^{-1}$, with superior capacity retention $(73.92 \%$ after 2,000 cycles at $\left.100 \mathrm{mAg}^{-1}\right)$. The findings reported here are beneficial for the design and manufacture of rechargeable sodium-ion batteries, positioning EG as a promising anodic material.

\section{Methods}

GO and EG synthesis. GO was synthesized through a modified Hummer's method $^{13}$. At room temperature, $1 \mathrm{~g}$ of graphite (synthetic, Sigma-Aldrich) and $0.5 \mathrm{~g}$ of $\mathrm{NaNO}_{3}$ (reagent plus, Sigma-Aldrich) were added into $23 \mathrm{ml}$ concentrated sulphuric acid (95-98\%, ACS reagent, Sigma-Aldrich) in a round-bottomed flask. The flask was then put into an ice bath. Three grams of $\mathrm{KMnO}_{4}$ (ACS reagent, Sigma-Aldrich) was added into the mixture slowly while stirring, during which the temperature was well controlled to lower than $20^{\circ} \mathrm{C}$. Then the temperature was brought to $35^{\circ} \mathrm{C}$ and maintained for $2 \mathrm{~h}$. Forty-six millilitres of room temperature $\mathrm{H}_{2} \mathrm{O}$ was added into the mixture slowly. The temperature was well controlled to below $98^{\circ} \mathrm{C}$ during this process. After mixing, the temperature was then slowly brought up to $98^{\circ} \mathrm{C}$ and maintained for $30 \mathrm{~min}$. A quantity of $140 \mathrm{ml}$ of warm water was further added to the liquid, followed by $20 \mathrm{ml}$ of $\mathrm{H}_{2} \mathrm{O}_{2}\left(30 \mathrm{wt} \%\right.$ in $\mathrm{H}_{2} \mathrm{O}$, Sigma-Aldrich), upon which golden particles and dark brown solid particles were formed. The mixture was filtered while still warm. Around 1 litre of warm $\mathrm{H}_{2} \mathrm{O}$ was further used to wash the solid until the supernatant was $\mathrm{pH}$ neutral at the end of washing. The resulting dark brown solid was collected as the GO product. To synthesize EG, the fore-mentioned GO was reduced by heat treatment. GO powder was put into alumina combustion boat in tube furnace with a continuous flow of Ar. The temperature was then brought from 25 to $600{ }^{\circ} \mathrm{C}$ with a heating rate of $5^{\circ} \mathrm{C} \mathrm{min}-1$. Then, the temperature was maintained at $600{ }^{\circ} \mathrm{C}$ for 1 and $5 \mathrm{~h}$ to reach different degrees of reduction.

Material characterization. The morphologies and microstructures of PG, GO and EG were characterized by a JEOL JEM-2100F TEM operated at $200 \mathrm{kV}$. For general structural characterization, the graphitic materials were transferred onto copper grids with amorphous carbon film support after stirring in isopropanol. For the XRD tests (Supplementary Fig. 1), the sample was scanned from $5^{\circ}$ to $90^{\circ}$ at a scan rate of $0.5^{\circ}$ per second in non-spin mode. The XRD data were collected by a D8 Advance diffractometer with LynxEye and SolX detectors in the X-ray Crystallographic Center at the University of Maryland. Brunauer-Emmett-Teller test was carried out on the Tristar II 3020 surface analysis system. In a typical measurement, $\sim 100 \mathrm{mg}$ of the powder sample was first degased for $5 \mathrm{~h}$, and then loaded to the system for measurement. In a typical tap density test, $\sim 1 \mathrm{~g}$ of the sample was put into a graduated measuring cylinder and then tapped for 50 times until no further volume change was observed. The volume was obtained by reading the inner diameter of the cylinder. The electronic conductivity of the samples was measured by four-probe method. In a typical measurement, powder sample with $\sim 0.1 \mathrm{~g}$ in mass was assembled in a Swagelok cell with an inner diameter of $13 \mathrm{~mm}$. The assembled cell was then loaded for $50 \mathrm{Mpa}$ pressure for $3 \mathrm{~min}$ to achieve the pallets. The pallet samples were then measured using a four-probe method (Signatone SP4) at University of Maryland Nanocenter.

Typical Raman tests (Supplementary Fig. 2c) were carried out over the dried powder samples. For the ex situ Raman characterization (Supplementary Fig. 7), in a typical procedure, EG was assembled into a home-made glass cell with airtight 
structure. The cell was assembled with an EG working electrode, a sodium metal counter electrode and $1.0 \mathrm{M} \mathrm{NaClO}_{4}$ in polycarbonate solvent liquid electrolyte in glove box with $\mathrm{Ar}$ atmosphere. Raman data were collected by Horiba-Jobin-Yvon Raman microscope in the University of Maryland Nanocenter with laser wavelength of $532 \mathrm{~nm}$.

XPS investigation was performed on powdered samples at room temperature. Data were collected using a high-sensitivity Kratos AXIS 165 spectrometer with survey pass energy of $160 \mathrm{eV}$ and high-resolution pass energy of $20 \mathrm{eV}$, also at the University of Maryland Nanocenter.

The solid-state NMR experiments were performed at ${ }^{1} \mathrm{H}$ frequencies of $500.16 \mathrm{MHz}$ with Bruker Avance III system using a Bruker 3.2-mm tripleresonance E-free MAS probe in a double resonance mode. ${ }^{13} \mathrm{C}$ MAS spectra in Fig. 4 were collected by a rotor-synchronous echo sequence with (Fig. 4a) and without (Fig. 4 b,c) a background suppression pulse sequence ${ }^{35}$ using a Kel-F cap. The background suppression sequence was used in Fig. $4 \mathrm{~b}, \mathrm{c}$ to quantify the signals of very limited oxidized species without the effects of background signals. In the ${ }^{13} \mathrm{C}-{ }^{1} \mathrm{H}$ REDOR experiments ${ }^{36,37}$, the data were collected with ${ }^{13} \mathrm{C} \pi$-pulse sandwiched by ${ }^{13} \mathrm{C}-{ }^{1} \mathrm{H}$ dephasing periods of four rotor cycles $\left(\tau_{\mathrm{R}}=66.7 \mu \mathrm{s}\right)$. The details are described in the Supplementary Material.

Electrochemical characterization. Electrochemical properties were characterized using a half-cell against a pure Na counter electrode. To assemble a test cell, the EG powder was mixed with $15 \mathrm{wt} \%$ of Na alginate in water in mortar and milled for $30 \mathrm{~min}$. The resulting slurry was then cast on copper foil. After drying at room temperature for $\sim 12 \mathrm{~h}$, the sample was then transferred into a standard vacuum oven kept at $100^{\circ} \mathrm{C}$ for overnight. A 0.5 -inch circular die-punch was used to cut the electrode. The typical loading amount of active material was $\sim 0.5 \mathrm{mg} \mathrm{cm}^{-2}$. Coin cells, consisting of an EG electrode, a sodium metal counter electrode, Celgard 3501 separator and $1.0 \mathrm{M} \mathrm{NaClO}_{4}$ in polycarbonate solvent liquid electrolyte, were assembled in a glove box under Ar atmosphere for further electrochemical tests.

Galvanostatic charge/discharge was carried out on an Arbin BT-2000 test station. Cells were cycled between 0.0 and $2.0 \mathrm{~V}$ (versus $\mathrm{Na} / \mathrm{Na}^{+}$) at different current densities. After the cell reached the cutoff voltage, it was relaxed for $10 \mathrm{~min}$ before subsequent charge or discharge. The capacity was calculated based on the mass of active material.

For the rate capability tests, cells were pre-cycled for 20 cycles for activation and then charged-discharged at different current densities between 0.0 and $2.0 \mathrm{~V}(\mathrm{Na} /$ $\mathrm{Na}^{+}$). The capacity is calculated based on the mass of EG.

The cyclic voltammetry test was carried out on the Solartron 1260/1287 Electrochemical Interface test station (Solartron Metrology, UK). Cells were precycled for 20 cycles to achieve the stable status before loaded to the testing system. The scan range was $0-2 \mathrm{~V}$ (versus $\mathrm{Na} / \mathrm{Na}^{+}$) with various scan rates.

HRTEM characterization. TEM characterization and in situ imaging were carried out on a JEOL $2100 \mathrm{~F}$ transmission electron microscope operated at $200 \mathrm{kV}$. Regular HRTEM observation was conducted for ex situ samples to determine their structures and morphologies at different conditions. In situ HRTEM experiments were performed with a customized NanoFactory manipulation holder equipped with a piezo-driven Pt probe. The setup is shown in Fig. 6a. In a typical experiment, EG was glued onto a specimen rod using conductive Ag epoxy, and Na metal was scratched off a fresh-cut surface of Na bulk using the Pt manipulator inside a glove box filled with high-purity nitrogen to prevent oxidation. Then the Na metal was intentionally exposed to the air for $<5 \mathrm{~s}$ during the holder loading process, in order to form a thin layer of $\mathrm{Na}_{2} \mathrm{O}$ on the surface, serving as a solid electrolyte. During the in situ operation, potentials of $-1.0 \mathrm{~V}$ to $-4.0 \mathrm{~V}$ were applied to EG with respect to $\mathrm{Na}$ metal to initiate sodiation, and $+1.0 \mathrm{~V}$ to $+4.0 \mathrm{~V}$ for desodiation.

\section{References}

1. Molenda, J., Delmas, C. \& Hagenmuller, P. Electronic and electrochemical properties of $\mathrm{Na}_{\mathrm{x}} \mathrm{CoO}_{2-\mathrm{y}}$ cathode. Solid State Ionics 9\&10, 431-435 (1983).

2. Nagelberg, A. S. \& Worrel, W. L. A thermodynamic study of sodiumintercalated $\mathrm{TaS}_{2}$ and $\mathrm{TiS}_{2}$. J. Solid State Chem. 29, 345-354 (1979).

3. Delmas, C., Braconnier, J.-J., Fouassier, C. \& Hagenmuller, P. Electrochemical intercalation of sodium in $\mathrm{Na}_{\mathrm{x}} \mathrm{CoO}_{2}$ bronzes. Solid State Ionics 3-4, 165-169 (1981).

4. Ge, P. \& Fouletier, M. Electrochemical interaction of sodium in graphite. Solid State Ionics 28-30, 1172-1175 (1988).

5. Stevens, D. A. \& Dahn, J. R. The mechanisms of lithium and sodium insertion in carbon materials. J. Electrochem. Soc. 148, A803-A811 (2001).

6. Cao, Y. et al. Sodium ion insertion in hollow carbon nanowires for battery applications. Nano Lett. 12, 3783-3787 (2012).

7. Zhao, J. et al. Electrochemical and thermal properties of hard carbon-type anodes for Na-ion batteries. J. Power Sources 244, 752-757 (2013).

8. Ponrouch, A., Goñi, A. R. \& Palacín, M. R. High capacity hard carbon anodes for sodium ion batteries in additive free electrolyte. Electrochem. Commun. 27, 85-88 (2013).

9. Wenzel, S., Hara, T., Janek, J. \& Adelhelm, P. Room-temperature sodium-ion batteries: Improving the rate capability of carbon anode materials by templating strategies. Energy Environ. Sci. 4, 3342-3345 (2011).
10. Tang, K. et al. Hollow carbon nanosphere with superior rate capability for sodium-based batteries. Adv. Energy Mater. 2, 873-877 (2012).

11. Wang, Y.-X., Chou, S.-L., Liu, H.-K. \& Dou, S.-X. Reduced graphene oxide with superior cycling stability and rate capability for sodium storage. Carbon $\mathbf{5 7}$, 202-208 (2013).

12. Sangster, J. C-Na (carbon-sodium) system. J. Phase Equilib. Diff. 28, 571-579 (2007).

13. Hummers, Jr. W. S. \& Offeman, R. E. Preparation of graphitic oxide. J. Am. Chem. Soc. 80, 1339-1339 (1957).

14. Yagi, T., Utsumi, W., Yamakata, M.-a., Kikegawa, T. \& Shimomura, O. High-pressure in situ X-ray-diffraction study of the phase transformation from graphite to hexagonal diamond at room temperature. Phys. Rev. B 46, 6031-6039 (1992).

15. Aurbach, D. \& Ein-Eli, D. The Study of Li-graphite intercalation processes in several electrolyte systems using in situ X-ray diffraction. J. Electrochem. Soc. 142, 1746-1752 (1995).

16. Lerf, A., He, H., Forster, M. \& Klinowski, J. Structure of graphite oxide revisited. J. Phys. Chem. B 102, 4477-4482 (1998).

17. Szabó, T. et al. I. Evolution of surface functional groups in a series of progressively oxidized graphite oxides. Chem. Mater. 18, 2740-2749 (2006)

18. Xu, B. et al. What is the choice for supercapacitors: graphene or graphene oxide? Energy Environ. Sci. 4, 2826-2830 (2011).

19. Sing, K. S. W. Reporting physisorption data for gas/solid systems with special reference to the determination of surface area and porosity. Pure Appl. Chem 57, 603-619 (1985)

20. Ng, S. H. et al. Correlations between surface properties of graphite and the first cycle specific charge loss in lithium-ion batteries. Carbon 47, 705-712 (2009).

21. Xing, W., Xue, J. S. \& Dahn, J. R. Optimizing pyrolysis of sugar carbons for use as anode materials in lithium-ion batteries. J. Electrochem. Soc. 143, 3046-3052 (1996).

22. Zheng, T., Xue, J. \& Dahn, J. R. Lithium insertion in hydrogen-containing carbonaceous materials. Chem. Mater. 8, 389-393 (1996).

23. Zheng, T., Zhong, Q. \& Dahn, J. R. High-capacity carbons prepared from phenolic resin for anodes of lithium-ion batteries. J. Electrochem. Soc. 142, L211-L214 (1995).

24. Wang, Y., Alsmeyer, D. C. \& McCreery, R. L. Raman spectroscopy of carbon materials: structural basis of observed spectra. Chem. Mater. 2, 557-563 (1990).

25. Cai, W. et al. Synthesis and solid-state NMR structural characterization of ${ }^{13} \mathrm{C}$-labeled graphite oxide. Science 321, 1815-1817 (2008).

26. Casabianca, L. B. et al. NMR-based structural modeling of graphite oxide using multi-dimensional ${ }^{13} \mathrm{C}$ solid-state NMR and ab-initio chemical shift calculations. J. Am. Chem. Soc. 132, 5672-5676 (2010).

27. Gao, W., Alemany, L. B., Ci, L. J. \& Ajayan, P. M. New insights into the structure and reduction of graphite oxide. Nat. Chem. 1, 403-408 (2009).

28. DiVincenzo, D. P. \& Mele, E. J. Cohesion and structure in stage-1 graphite intercalation compounds. Phys. Rev. B 32, 2538-2553 (1985).

29. Gotoh, K. et al. NMR study for electrochemically inserted $\mathrm{Na}$ in hard carbon electrode of sodium ion battery. J. Power Sources 225, 137-140 (2013).

30. Stevens, D. A. \& Dahn, J. R. High capacity anode materials for rechargeable sodium-ion batteries. J. Electrochem. Soc. 147, 1271-1273 (2000).

31. Shao, Y. et al. Surface-driven sodium ion energy storage in nanocellular carbon foams. Nano Lett. 13, 3909-3914 (2013).

32. Augustyn, V. et al. High-rate electrochemical energy storage through $\mathrm{Li}^{+}$ intercalation pseudocapacitance. Nat. Mater. 12, 518-522 (2013).

33. Ardizzone, S, Fregonara, G \& Trasatti, S. 'Inner' and 'outer' active surface of $\mathrm{RuO}_{2}$ electrodes. Electrochim. Acta 35, 263-267 (1990).

34. Mochida, I., Ku, C.-H., Yoon, S.-H. \& Korai, Y. Anodic performance and mechanism of mesophase-pitch-derived carbons in lithium ion batteries. J. Power Sources 75, 214-222 (1998).

35. Chen, Q., Hou, S. S. \& Schmidt-Rohr, K. A simple scheme for probehead background suppression in one-pulse ${ }^{1} \mathrm{H}$ NMR. Solid State Nucl. Magn. Reson. 26, 11-15 (2004).

36. Ishii, Y., Wickramasinghe, N. P. \& Chimon, S. A new approach in 1D and 2D ${ }^{13} \mathrm{C}$ high resolution solid-state NMR spectroscopy of paramagnetic organometallic complexes by very fast magic-angle spinning. J. Am. Chem. Soc. 125, 3438-3439 (2003).

37. Wickramasinghe, N. P. et al. Progress in ${ }^{13} \mathrm{C}$ and ${ }^{1} \mathrm{H}$ solid-state NMR for paramagnetic systems under very fast magic angle spinning. J. Chem. Phys. 128, 052210-1-052210-15 (2008).

\section{Acknowledgements}

This work was supported as part of the Science of Nanostructures for Electrical Energy Storage, an Energy Frontier Research Center funded by the US Department of Energy, Office of Science, Office of Basic Energy Sciences under Award Number DESC0001160. We also acknowledge funding for the NMR studies by Y.I. (NSF CHE-1310363) and the use of facilities in Maryland NanoCenter and its NISP Lab supported in part by the NSF 
MRSEC under grant DMR 05-20471. We thank Dr Karen Gaskell at the Surface Analysis Center of University of Maryland for the help on the XPS data analysis.

\section{Author contributions}

Y.W., Y.X. and C.W. conceived the ideas; Y.W. prepared the materials and performed majority of the materials characterization and electrochemical tests; F.H. carried out the electronic conductivity test; K.H. and Y.W. carried out the in situ TEM experiments under the direction of J.C. and C.W., respectively; I.M. and Y.I. performed the MASNMR characterization, and co-wrote the related session; Y.W. and Y.Z. analysed all the data; and Y.W., K.H. and Y.Z. co-wrote the manuscript. All authors discussed and commented on the manuscript.

\section{Additional information}

Supplementary Information accompanies this paper at http://www.nature.com/ naturecommunications

Competing financial interests: The authors declare no competing financial interests.

Reprints and permission information is available online at http://npg.nature.com/ reprintsandpermissions/

How to cite this article: Wen, Y. et al. Expanded graphite as superior anode for sodium-ion batteries. Nat. Commun. 5:4033 doi: 10.1038/ncomms5033 (2014). 